\title{
Manejo anestésico en paciente con síndrome de Brugada, a propósito de un caso
}

\section{Case report: Anesthesia for a patient with Brugada syndrome}

\author{
Pablo Arco de la Torre ${ }^{1, *}$, María del Pilar Sáenz Pascual ${ }^{1}$, Marta Aguado Sevilla1 \\ 1 Hospital San Pedro. Logroño, España.
}

Fecha de recepción: 18 de abril de 2021 / Fecha de aceptación: 30 de abril de 2021

\begin{abstract}
Brugada syndrome (BS) is a rare entity, with an estimated prevalence of approximately $1: 5,000$. It is present in $4 \%$ of all sudden deaths and up to $20 \%$ of sudden deaths in patients without structural heart defects. Recognition of the electrocardiographic pattern is crucial, since the 2-year mortality in Brugada syndrome is close to $30 \%$. The Brugada pattern becomes more pronounced when vagal activity is predominant, for example, during sleep, hence the sudden nocturnal death syndrome. Anesthetic management in these patients must be very careful, therefore we present the case of a patient with Brugada syndrome scheduled for varicose vein surgery. We expose the considerations and anesthetic management that was carried out.
\end{abstract}

Key words: Brugada sindrome, subaracnoid anesthesia.

\section{RESUMEN}

El síndrome de Brugada (SB) es una entidad poco común, con una prevalencia estimada de aproximadamente 1:5.000. Está presente en el $4 \%$ de todas las muertes súbitas y hasta en el $20 \%$ de las muertes súbitas en pacientes sin defectos cardíacos estructurales. El reconocimiento del patrón electrocardiográfico es crucial, dado que la mortalidad a 2 años en el síndrome de Brugada es cercana al 30\%. El patrón de Brugada se hace más pronunciado cuando la actividad vagal es predominante, por ejemplo, durante el sueño, de ahí el síndrome de muerte súbita nocturna. El manejo anestésico en estos pacientes debe ser muy cuidadoso, por ello presentamos el caso de un paciente con síndrome de Brugada programado para cirugía de varices. Exponemos las consideraciones y manejo anestésico que se llevó a cabo.

Palabras clave: Síndrome de brugada, anestesia subaracnoidea.

\section{Introducción}

E I síndrome de Brugada fue descrito por primera vez en 1992 y se caracteriza por la presencia de un patrón electrocardiográfico de bloqueo de rama derecha con elevación del ST en las derivaciones V1 a V3 y un intervalo QT normal, además, de una predisposición a la presencia de arritmias ventriculares y muerte súbita[1]. Tiene un componente hereditario de tipo autosómico dominante debido a la mutación del gen SNC5A del cromosoma 3 que codifica los canales de conductancia del sodio y se engloba dentro de las canalopatías que característicamente no asocian cardiopatía estructural concomitante.

A nivel anestésico se ha visto que determinados factores, tales como la hipertermia, los cambios autonómicos o el uso de determinados fármacos, pueden desencadenar crisis de arritmias, lo que nos lleva a realizar una monitorización cautelosa de estos pacientes en el intraoperatorio y a evitar los posibles causantes de eventos arritmogénicos[2].

\section{Descripción del caso}

Presentamos el caso de un paciente de 40 años sometido 
a cirugía de varices en miembro inferior derecho. Alérgico a la aspirina y sin otros antecedentes de interés, fue diagnosticado de síndrome de Brugada tipo I en el año 2007 a la edad de 27 años tras la muerte súbita de un familiar de segundo grado, se le realizó un estudio electrofisiológico que fue normal y portó un holter subcutáneo durante dos años que no registró eventos arrítmicos. En la consulta preanestésica se objetivó en el electrocardiograma un patrón de Brugada tipo I (Figuras 1 y 2) y también fue valorado por el servicio de cardiología, que constató la ausencia de clínica a nivel cardiovascular sin síncopes previos ni cardiopatía estructural mediante ecocardiografía transtorácica. En el antequirófano, se habla con el paciente explicándole los posibles riesgos y complicaciones cardiológicas en función de los anestésicos empleados para la cirugía.

Al llegar a quirófano, se monitoriza al paciente mediante electrocardiograma, pulsioximetría y presión arterial no invasiva y se dispone de desfibrilador externo junto a él. Posteriormente, se coloca al paciente en decúbito lateral derecho (por mala tolerancia a la sedestación y episodio de bradicardia sinusal de origen vagal), se administra atropina $0,7 \mathrm{mg}$ y se realiza anestesia subaracnoidea a nivel L3-L4 con 36 mg de prilocaína hiperbárica al 2\% más 10 microgramos de fentanilo[3] con punción única, atraumática, mediante una aguja espinal punta de lápiz $27 \mathrm{G}$ Whitacre ${ }^{\circledR}$. Se realiza sedación con midazolam 6 mg intravenosos y se le administra oxígeno a través de gafas nasales a $2 \mathrm{l} / \mathrm{min}$. Como analgesia y antiemésis se administra un gramo de paracetamol endovenoso y $4 \mathrm{mg}$ de ondansetrón 15 minutos antes de finalizar la intervención. Durante todo el procedimiento se mantiene hemodinámicamente estable con presiones arteriales medias en torno a $70 \mathrm{mmHg}$, frecuencias cardíacas entre 55-60 latidos/minuto con el patrón Brugada tipo I ya conocido y buenas saturaciones periféricas de oxígeno. La intervención transcurre sin incidencias y es trasladado a la Unidad de Recuperación posanestésica (URPA), donde recupera la movilidad y sensibilidad de miembros inferiores quince minutos después de la realización de la técnica neuroaxial. Tras $24 \mathrm{~h}$ de posoperatorio y ante la buena evolución se traslada a planta para continuidad de cuidados. Se mantiene estable a nivel cardiológico durante todo el ingreso y es dado de alta a su domicilio al día siguiente por parte de cirugía vascular.

\section{Discusión}

El empleo de anestesia en pacientes con síndrome de Brugada ya sea general o regional, ha supuesto siempre un reto para el anestesiólogo[4]. Hay que tener especial cuidado con los posibles factores desencadenantes de arritmias tales como determinados fármacos (se detallan después), episodios de bradicardia, variaciones térmicas, hiper o hipopotasemia.

De entre los fármacos de uso habitual en anestesia las benzodiacepinas no han sido asociados con eventos adversos.

Respecto a los anestésicos endovenosos, el uso del propofol sigue siendo controvertido, está en las listas de fármacos a evitar si bien con un grado de evidencia lla y aquellos estudios que muestran patrones electrocardiográficos de Brugada se han asociado con infusiones prolongadas de propofol (síndrome de infusión de propofol). En otros estudios de series de casos el propofol ha sido usado para la inducción en bolus sin incidencias. El tiopental y el etomidato han sido utilizados como induc-

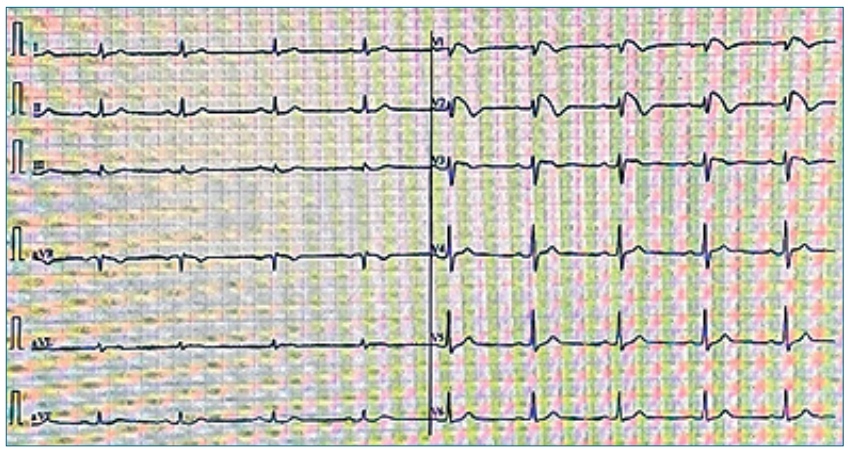

Figura 1.

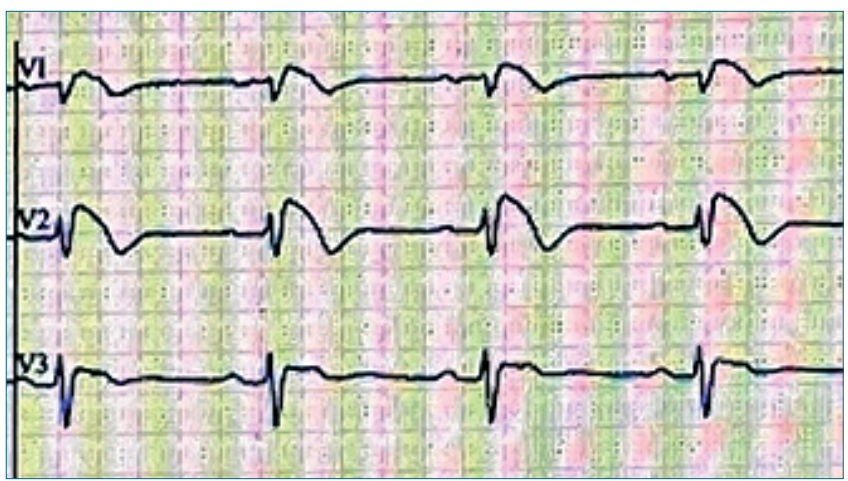

Figura 2.

tores sin incidencias, salvo algún caso autolimitado de elevación del ST con el etomidato.

El uso de los anestésicos inhalatorios parece ser más seguro. El isofluorano prolonga el intervalo QTc mientras que el halotano lo acorta. Se ha podido efectuar anestesia general sin complicaciones con óxido nítrico, desfluorano, isofluorano y sevofluorano, siendo este último de elección.

En cuanto a los anestésicos locales, la anestesia subaracnoidea se ha realizado con éxito usando lidocaína, mepivacaína, bupivacaína y ropivacaína. Destacar que los anestésicos locales son bloqueantes de los canales de sodio y que su potencia de bloqueo varía de unos a otros. La bupivacaína produce cambios más prolongados en la despolarización y su isómero $\mathrm{R}(+)$ bloquea los canales de sodio con gran potencia y se disocia lentamente, esto lo transforma en el anestésico local de mayor potencial proarrítmico y cardiotóxico. Hay casos reportados de patrón de Brugada inducido por la aplicación de bupivacaína epidural.

En caso de aplicar anestesia neuroaxial en pacientes con síndrome de Brugada, la primera opción debería ser un anestésico de acción corta y en caso de utilizar anestésicos de acción prolongada, la dosis total debería ser la mínima posible[5].

Respecto a los opioides en su gran mayoría no han presentado complicaciones, sin embargo, se deberá tener en cuenta su efecto bradicardizante para evitar la aparición de complicaciones. Preferiblemente se deberían utilizar opioides de acción corta o ultracorta por su rápida eliminación. En el posoperatorio es preferible la morfina (estudios publicados demuestran que el tramadol, fentanilo y sufentanilo bloquean con más potencia 
los canales de sodio que la morfina). El tramadol ha sido documentado como posible causante de aparición de patrón de Brugada, aunque con un grado de evidencia llb.

Sobre los relajantes neuromusculares y sus reversores, la succinilcolina no ha presentado efectos adversos en varios casos, pero en el trabajo de Kloesel hubo pacientes con elevación del ST después de recibir dosis de inducción con este relajante[6]. En adultos ocurre típicamente una bradicardia cuando se repite la dosis de succinilcolina en un segundo bolo que se administre entre 3 y 8 minutos después de la primera dosis (un metabolito de la succinilcolina, la succinilmonocolina, sensibiliza los receptores colinérgicos muscarínicos en el nódulo sinoauricular a esta segunda dosis, lo que resulta en bradicardia). Los relajantes neuromusculares no despolarizantes rocuronio, vecuronio, atracurio, cisatracurio y mivacurio han sido todos usados sin presentar efectos adversos. Respecto a la neostigmina, la estimulación parasimpática que provoca puede causar cambios en el ST en el síndrome de Brugada y su uso es controvertido. El uso de sugammadex como reversor es una buena opción cuando se trate de los bloqueantes neuromusculares no despolarizantes esteroideos (rocuronio o vecuronio)[7].

\section{Conclusiones}

La anestesia, ya sea general o regional, de los pacientes con síndrome de Brugada precisa de una vigilancia y monitorización continua de la frecuencia cardíaca, el trazado electrocardiográfico, la tensión arterial y en caso de anestesia general, de la profundidad anestésica, temperatura corporal y bloqueo neuromuscular.

La elección de los fármacos anestésicos viene dada por sus propiedades sobre el sistema nervioso autónomo y la evidencia científica. La presencia de un desfibrilador automático en qui- rófano es imprescindible por ser el único tratamiento efectivo frente a las arritmias ventriculares en el síndrome de Brugada.

Es necesaria la vigilancia posoperatoria estrecha tanto de la frecuencia cardíaca como del electrocardiograma, al menos durante las primeras $24 \mathrm{~h}$ tras la intervención para descartar la posible aparición de arritmias.

\section{Referencias}

1. Brugada J, Brugada R, Brugada P. Síndrome de bloqueo de rama derecha, elevación del segmento ST en las derivaciones V1 a V3 y muerte súbita cardiaca. Rev Esp Cardiol. 1998;51(3):169-70.

2. Carey SM, Hocking G. Brugada syndrome - A review of the implications for the anaesthetist. Anaesth Intensive Care. 2011;39 (4): 571-7.

3. B. Oliván, A. Arbeláez, M. de Miguel, A. Pelavski. Diagnóstico de síndrome de Brugada tras inyección subaracnoidea de prilocaína. Rev Esp Anestesiol Reanim, 63 (2016), pp. 483-486.

4. Espinosa A, Abad A, Rodríguez Bustamante V, Ripollés Melchor J, Marmanya Mezquita S, Izarduy L. Síndrome de Brugada y Anestesia: Generalidades. Rev Electron AnestesiaR [revista en Internet]. 2016 [citado 29 May 2016];8(3): [aprox. 9p]. Disponible en: http://anestesiar.org/rear/volumen-viii-2016/numero-03/664sindrome-de-brugada-generalidades.

5. Fernández Suárez FE, Argüelles Tamargo L, Varela Rodríguez L, Quintela Baizán AF. Síndrome de Brugada y anestésicos locales. Rev Esp Anestesiol Reanim. 2008;55(8):518.

6. Kloesel B, Ackerman MJ, Sprung J, et al. Anesthetic management of patients with Brugada syndrome: a case series and literature review. Can J Anaesth. 2011;58(9):824-836.

7. Conde R, Pereira M. Abordaje anestésico en paciente con el síndrome de Brugada - Uso del sugammadex en cirugía mayor abdominal. Rev Bras Anestesiol. 2013;63(1):159-60. 